\title{
Design and Fabrication of Wheelchair CUM Stretcher
}

\author{
Yash Shah, Neel Patel, Nischay Patel, Shail Patel, Piyush Surani
}

\begin{abstract}
The wheelchair and stretcher are very widely used in hospitals, airports, train stations, shopping malls, etc. This design here is a modified wheelchair stretcher as needed.This machine converts the wheelchair into a stretcher. The chair transforms into a stretcher when the levers are operated. The stretchers can be detached from the main frame according to the convenience of the patient and doctors can make it easier Access to the patient with less effort and transport The folding mechanism enables a large number of stretchers arranged in chair form to be accommodated in a comparatively smaller space. in hospitals, patients have to be moved from a wheelchair to a stretcher, from a stretcher to a bed, from a bed to a wheelchair or vice versa; which creates unsafe conditions for patients. A wheelchair stretcher is required to facilitate mobility for the disabled patient.
\end{abstract}

Keywords: Wheelchair, Stretcher, Space, Patient's Mobility.

\section{INTRODUCTION}

Theconcept of Design and Fabrication of Wheelchair cum Stretcher is useful for disabled patient's mobility.DISABILITY is the term that has been used for many decades. According to Oxford Etymology, "disability" is used in many contexts, namely 1650 as a sport called "CAP IN HAND", 1750 used in horse races, 1870 it is "Any race or competition in which the competitors try to equalize the odds, by giving an advantage to the less efficient or a disadvantage to the most efficient ". Finally, the first use of disability to designate a mental or physical disability is recorded in a caption from 1915: The disabled child. Since 1915 the word "DISABILITY / DISABILITY" has been the trade name for people with physical or mental disabilities.

\section{PROBLEM DEFINITION}

The proportion of patients in India is increasing day by day. In hospitals, patients have to move from the wheelchair to the stretcher, from the stretcher to the bed, from the bed to the wheelchair or vice versa; which creates unsafe conditions for patients. Moving patients to hospitals is a common problem for nurses.

Manuscript received on June 03, 2021.

Revised Manuscript received on June 09, 2021.

Manuscript published on June 30, 2021.

* Correspondence Author

Yash Shah*, UG Students, Mechanical Engineering, Indus University, Ahmedabad (Gujarat), India.

Neel Patel, UG Students, Mechanical Engineering, Indus University, Ahmedabad (Gujarat), India.

Nischay Patel, UG Students, Mechanical Engineering, Indus University, Ahmedabad (Gujarat), India.

Shail Patel, B.Tech, Mechanical Engineering, Indus University, Ahmedabad (Gujarat), India.

Piyush Surani, Professor, Mechanical Engineering, Indus University, Ahmedabad (Gujarat), India.

(c) The Authors. Published by Blue Eyes Intelligence Engineering and Sciences Publication (BEIESP). This is an open access article under the CC BY-NC-ND license (http://creativecommons.org/licenses/by-nc-nd/4.0/)

$y=\frac{d 1}{2}=\frac{10}{2}=5 \mathrm{~mm}$

$I=\frac{\pi}{64} * d_{1}^{4}=490.8739 \mathrm{~mm}^{4}$

$\sigma_{b}=79.13927 \frac{\mathrm{N}}{\mathrm{mm}^{2}}$

Shear Stress $=\tau=\frac{T R}{J}$

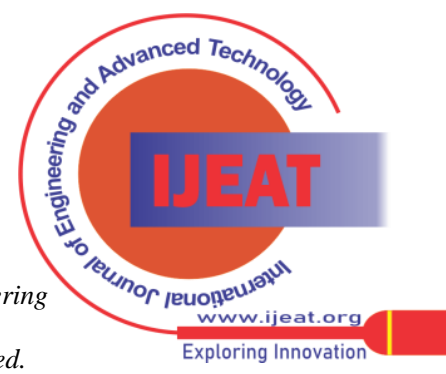


$\operatorname{Torque}(T)=F_{f 1} *\left(\frac{l}{2}\right)=388.474 * 20$

$$
=7769.48 \mathrm{~N} . \mathrm{mm}
$$

$R=\frac{d_{1}}{2}=5 \mathrm{~mm}$

$J=\frac{\pi}{32} d_{1}^{4}=981.7478 \mathrm{~mm}^{4}$

$\tau=39.56 \frac{\mathrm{N}}{\mathrm{mm}^{2}}$

Rear Wheel Stress:-

shaft Diameter $d_{2}$ Rear Wheel $=10 \mathrm{~mm}$

Stress $($ Bending $)=\sigma_{b}=\frac{M y}{I}$

$\operatorname{Moment}(2)($ Rear $)=F_{f 1} *\left(\frac{l}{2}\right)=388.474 * 50$ $=19423.7 \mathrm{~N} . \mathrm{mm}$

$y=\frac{d 1}{2}=\frac{10}{2}=5 \mathrm{~mm}$

$I=\frac{\pi}{64} * d_{1}^{4}=490.8739 \mathrm{~mm}^{4}$

$\sigma_{b}=197.848 \frac{\mathrm{N}}{\mathrm{mm}^{2}}$

Shear Stress $=\tau=\frac{T R}{J}$

$\operatorname{Torque}(T)=F_{f 1} *\left(\frac{l}{2}\right)=388.474 * 50$

$$
=19423.7 \mathrm{~N} . \mathrm{mm}
$$

$R=\frac{d_{1}}{2}=5 \mathrm{~mm}$

$J=\frac{\pi}{32} d_{1}^{4}=981.7478 \mathrm{~mm}^{4}$

$\tau=98.92 \frac{\mathrm{N}}{\mathrm{mm}^{2}}$

Factor of Safety:

F.O.S $=\frac{\text { Ultimate Tensile Stress on Material }}{\text { Maximum Stress Generated in Material }}$ $=\frac{500}{197.848}=2.52$

\section{3D DESIGN}

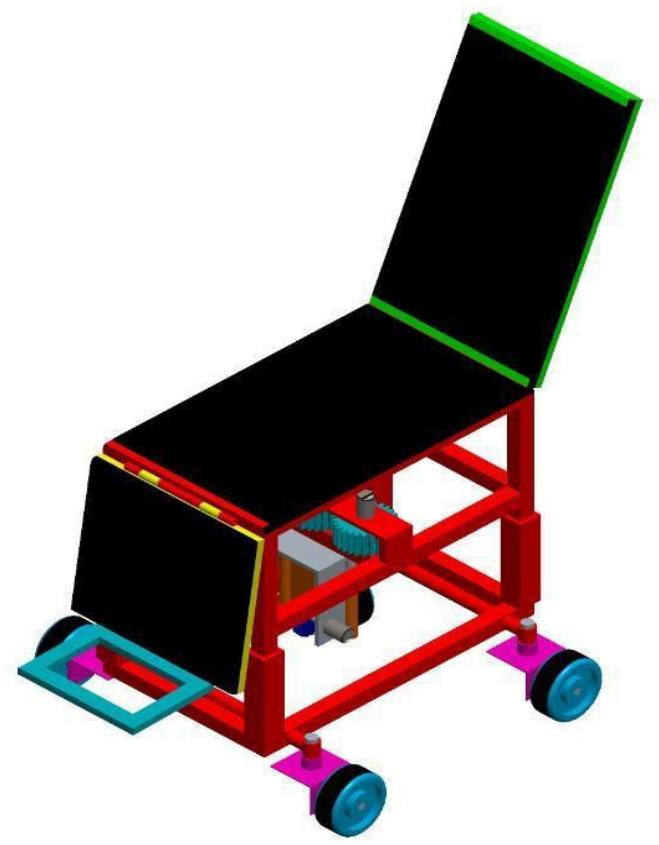

Retrieval Number: 100.1/ijeat.E27540610521
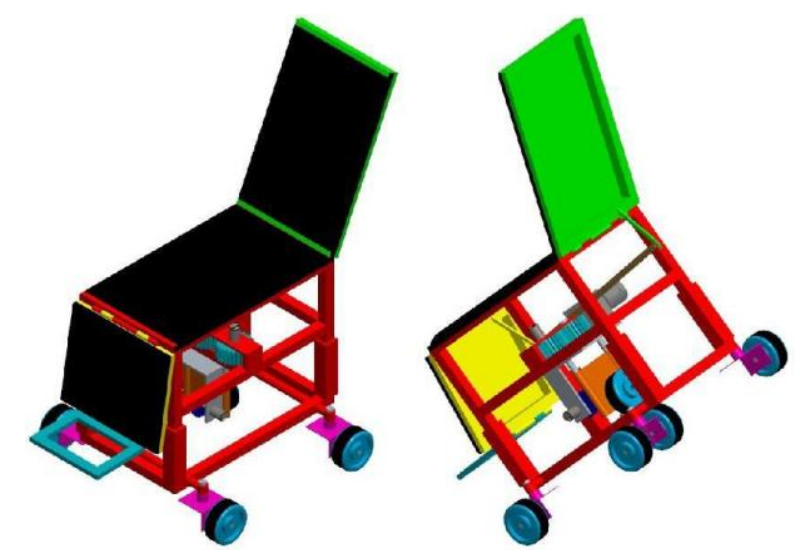

Back Frame Structure

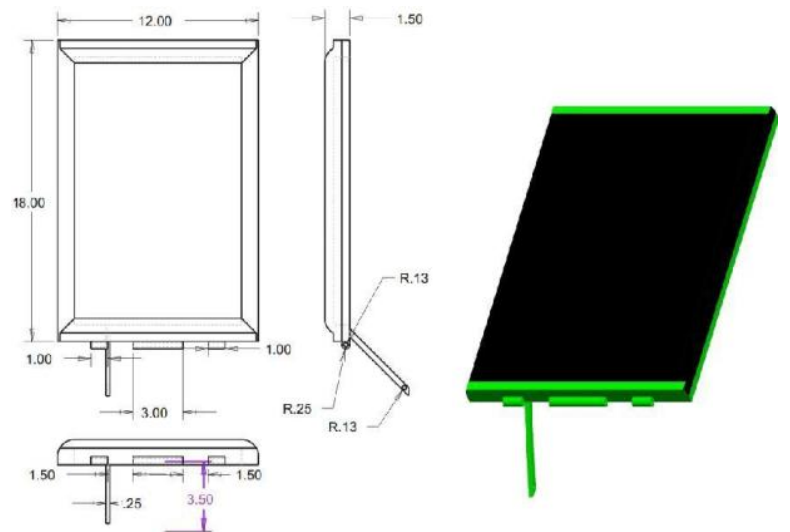

Center Frame Structure

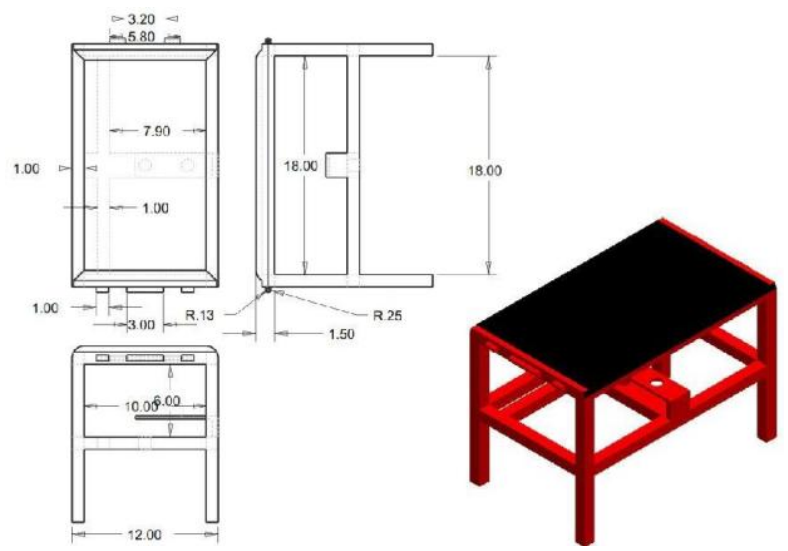

DC Motor
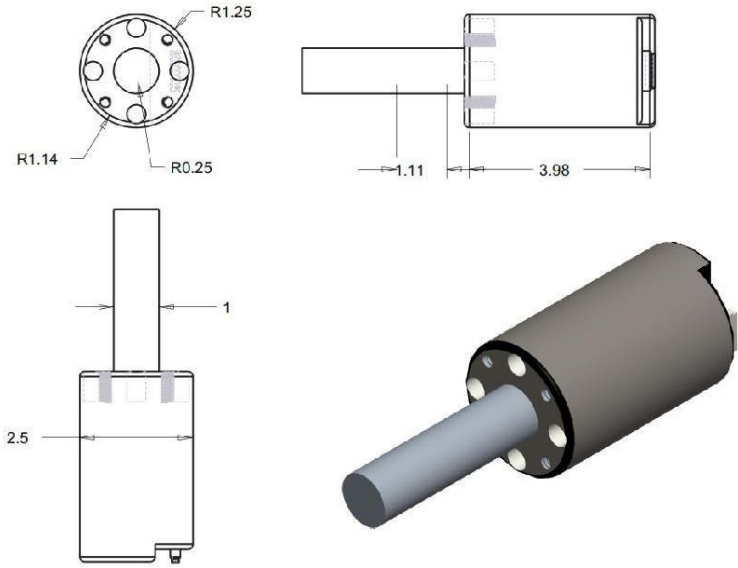

Leg Part -1

Published By:

Blue Eyes Intelligence Engineering and Sciences Publication

(C) Copyright: All rights reserved.

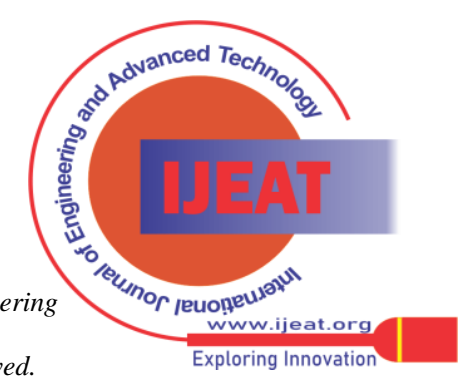




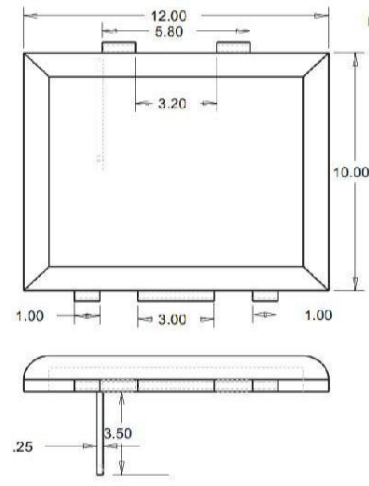

Leg Part -2
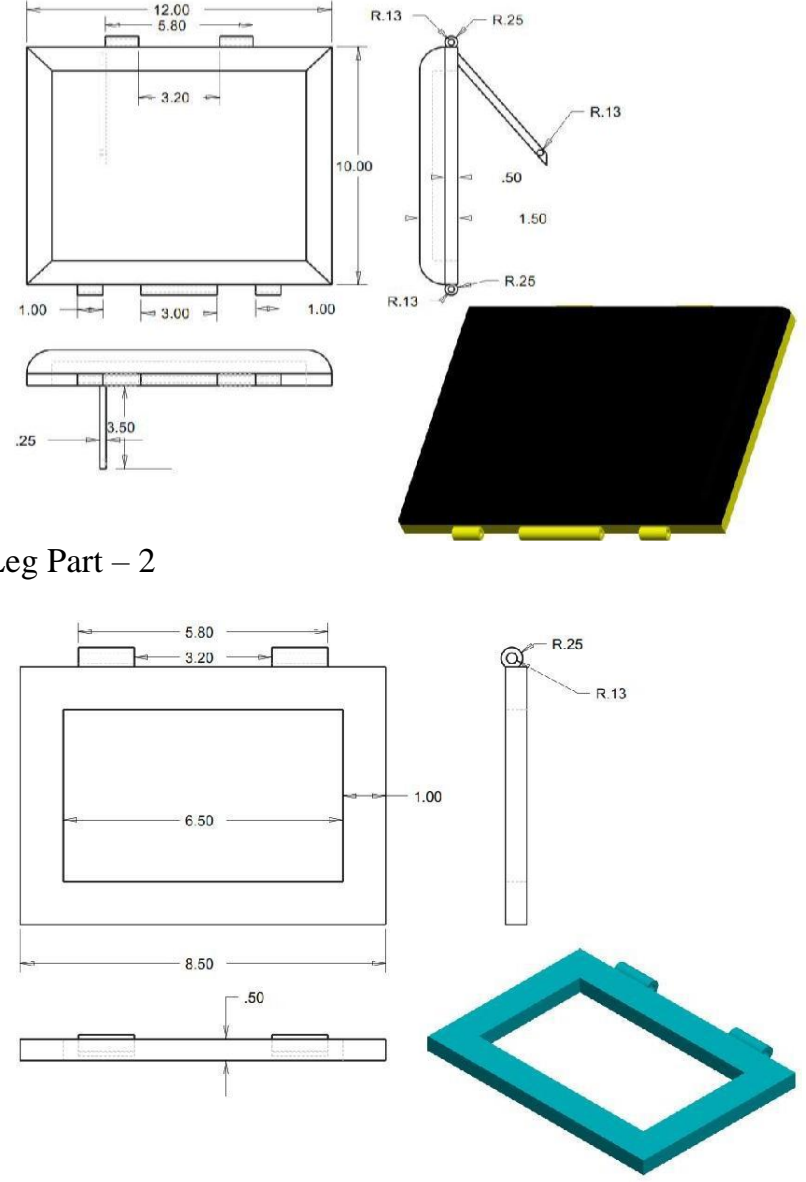

DC Motor High Torque
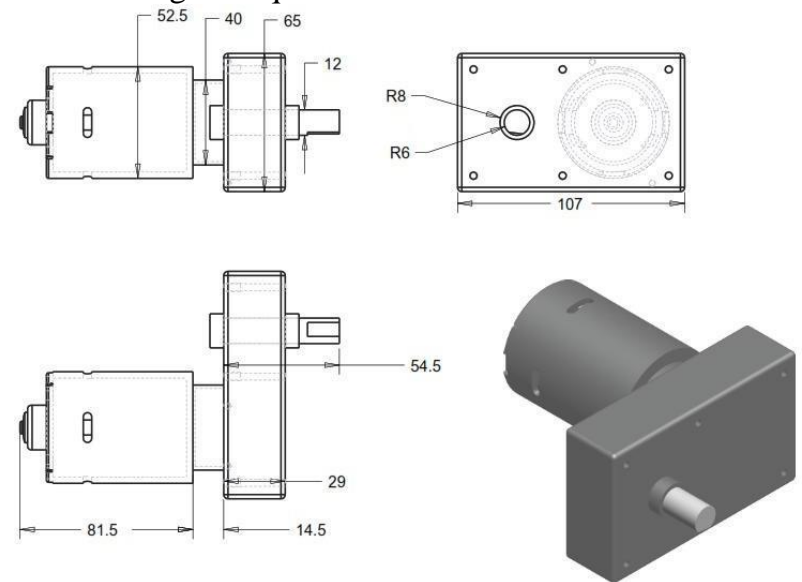

Wheel
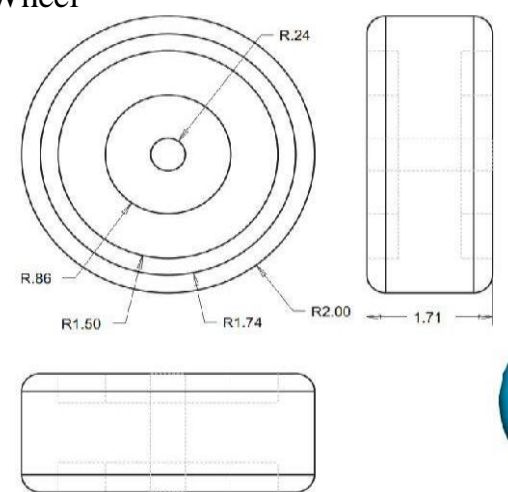

Slider

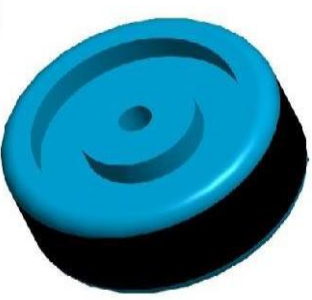

Retrieval Number: 100.1/ijeat E27540610521

DOI:10.35940/ijeat.E2754.0610521

Journal Website: www.ijeat.org
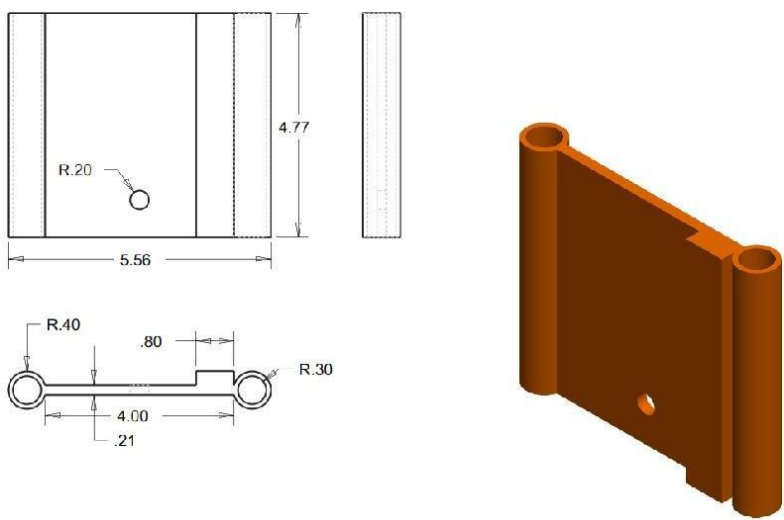

Slider Support
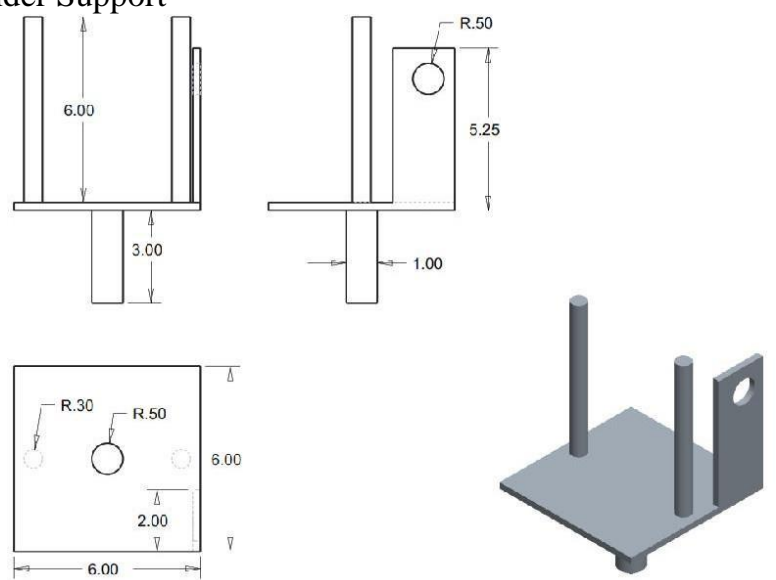

Rack

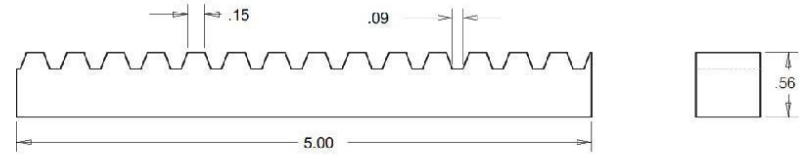

Pinion
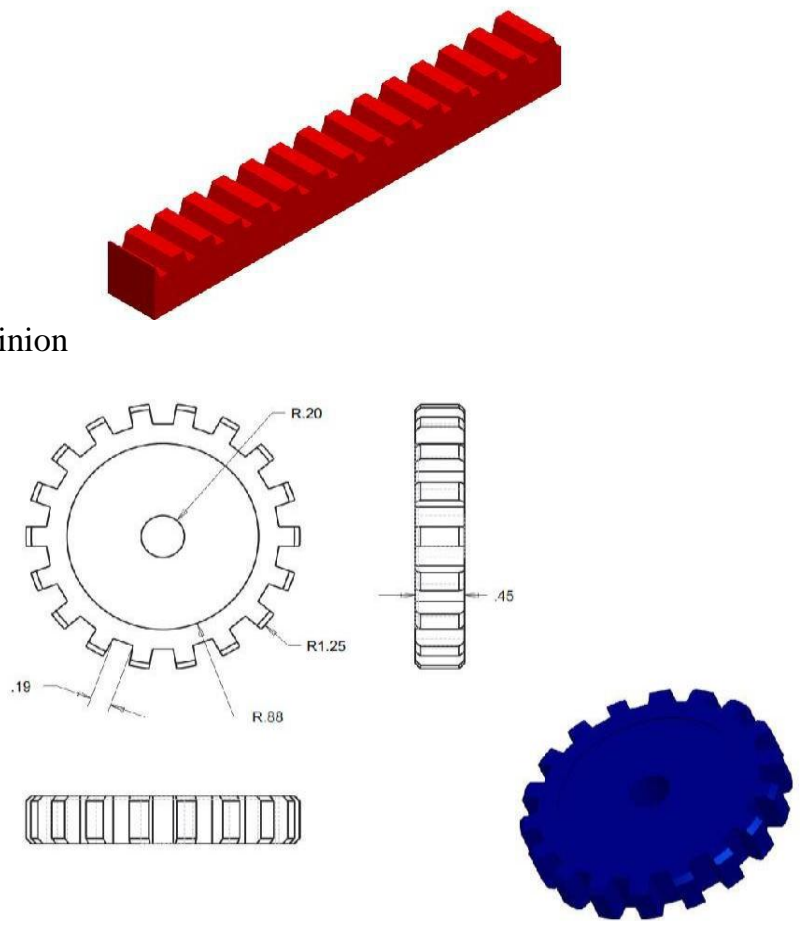

Small Gear

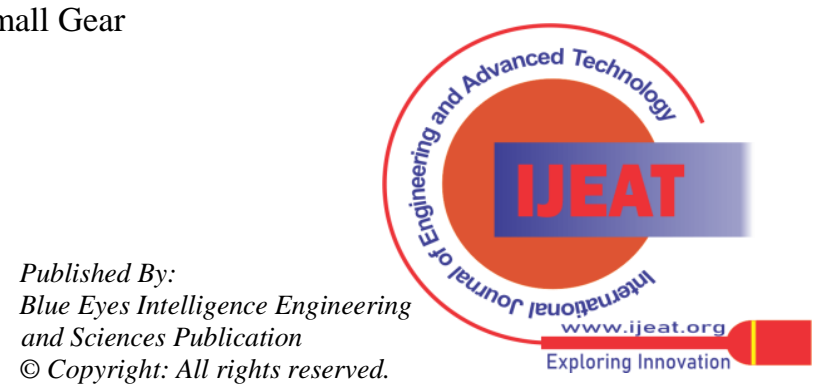




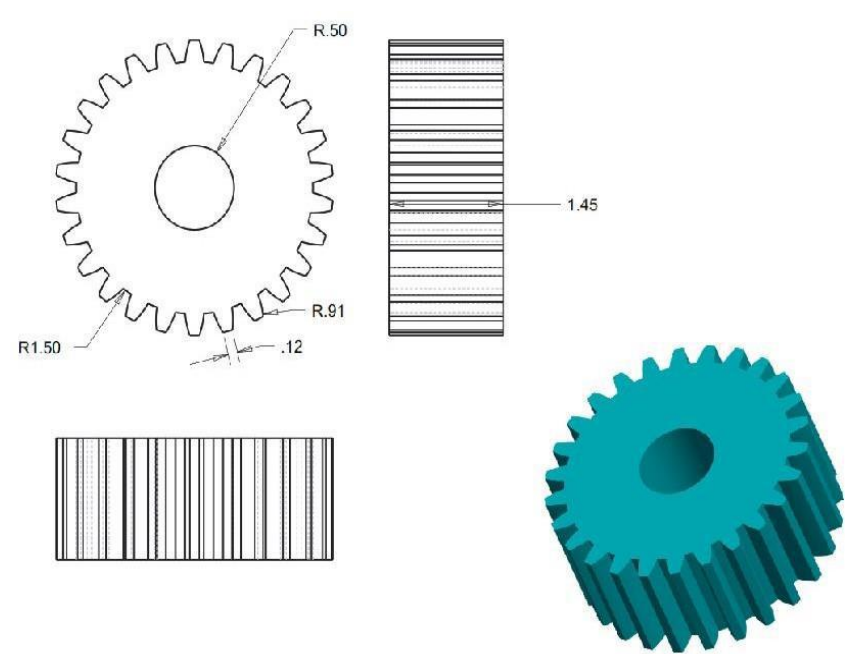

VI. CONNECTION GRAPH

Connection Graph of Transmitter

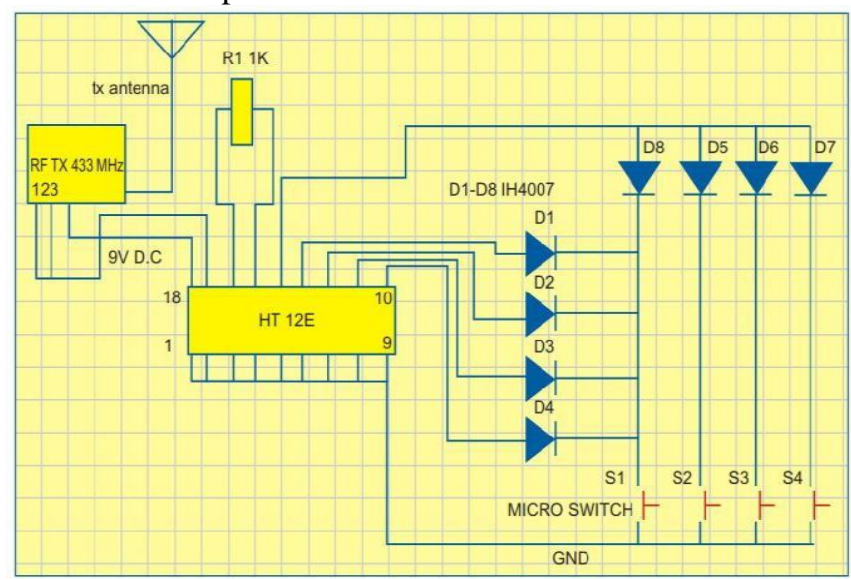

Connection Graph of Receiver

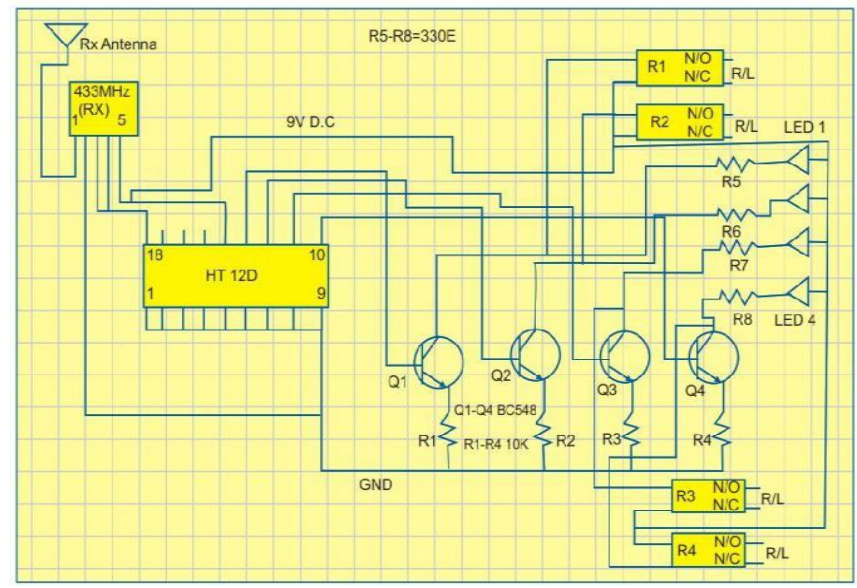

VII. LIST OF COMPONENT WITH ESTIMATION

\begin{tabular}{|c|l|c|c|c|}
\hline Sr.No & Part Name & $\begin{array}{c}\text { Quantity in } \\
\text { KG/Piece }\end{array}$ & Rate & $\begin{array}{c}\text { Amount } \\
\text { in RS }\end{array}$ \\
\hline $\mathbf{1}$ & SS Box Pipe & 30 feet & 80 & 2400 \\
\hline $\mathbf{2}$ & Seat & 1 Set & 3500 & 3500 \\
\hline $\mathbf{3}$ & Iron Rod & 3 feet & 100 & 300 \\
\hline $\mathbf{4}$ & Self Rotating Trolley Wheel & 2 nos & 400 & 800 \\
\hline $\mathbf{5}$ & Spring & 4 Nos & 100 & 400 \\
\hline $\mathbf{6}$ & Locking Nobs & 4 Nos & 50 & 200 \\
\hline $\mathbf{7}$ & 4 Inch HInges & 8 Nos & 150 & 1200 \\
\hline $\mathbf{8}$ & 4 Inch Rubber wheel & 2 Nos & 240 & 480 \\
\hline $\mathbf{9}$ & RF Remote & 1 Nos & 1900 & 1900 \\
\hline $\mathbf{1 0}$ & Wiper Motor & 1 nos & 1600 & 1600 \\
\hline $\mathbf{1 1}$ & Rack & 1 Nos & 1600 & 1600 \\
\hline $\mathbf{1 2}$ & Pinion & 1 Nos & 400 & 400 \\
\hline $\mathbf{1 3}$ & DC motor & 1 Nos & 3800 & 3800 \\
\hline $\mathbf{1 4}$ & wooden Plate & 1 nos & 600 & 600 \\
\hline $\mathbf{1 5}$ & DC Motor Mounting Clamp & 20 & 80 \\
\hline & & & 19260 \\
\hline & & & & \\
\hline
\end{tabular}

\section{REFERENCES}

1. Richard C. Simpson Phd, "Smart Wheelchairs",Department Of Rehabilitation Science And Technology, University OfPittsburgh,Pa(2005)

2. Sumedh. J. Suryawanshi, Dr. K. Janardhan Reddy "Product Development Of Wheelchair For People Disabled In Legs", (2013)(Smbs2013)

3. Roger Bostelman, James Albus"A Multipurpose Robotic Wheelchair And Rehabilitation Device For The Home” , (NistGaithersburg), (2007)

4. Prof.R.S.Nipanikar, Vinay Gaikwad, Chetan Choudhari, Ram Gosavi,VishalHarne"Automatic Wheelchair For Physically Disabled Persons", (2013) (Ijarece)

5. Prof Piyush Surani "Design And Development Of Conceptual wheelchair Cum Stretcher", Indus University (2021)

6. Mohan Kumar R., Lohit H. S., Manas Ranjan Mishra, Md. Basheer Ahamed, "Design Of Multipurpose Wheel Chair For Physically Challenged And Elder People" Department Of Design, M. S. Ramaiah School Of Advanced Studies, Bangalore.(2012)

7. Jingtao.Chen, Bing.Teng, Yali.Yang, "Design Of The Wheelchair Bed" Shanghai University Of Engineering Science Shanghai201620, China, (2013) •HuiHsu, Hsueh- Yu C1hen, JenYu Liu And Chien-Liang Chen, "Dual-Purpose Wheelchair Mechanism" Designs Meng, Proceedings of The International Multi conference Of Engineers And Computer Scientists 2009 Volli Imecs2009, March 18 -20, 2009, Hong Kong(2009)

8. Mst.NasimaBagum, Choudhury Abul AnamRashed,SanjoyKar,"Designing An Automated WheelChairWithStair Crossing Facility",International Journal Of Scientific\&EngineeringResearch,Volume3,Issue4,April-20121Issn22 295518,(2012)

9. Po Er Hsu ,Yeh Liang Hsu Jun Ming Lu1 And Cheng Hao Chang,"Seat Adjustment Design Of An Intelligent Robotic Wheelchair Based On The Stewart Platform" Regular Paper,Geron technology Research Center ,Yuan ZeUniversity Taoyuan, Taiwan,(2013)

Published By:
Blue Eyes Intelligence Engineering and Sciences Publication (c) Copyright: All rights reserved.

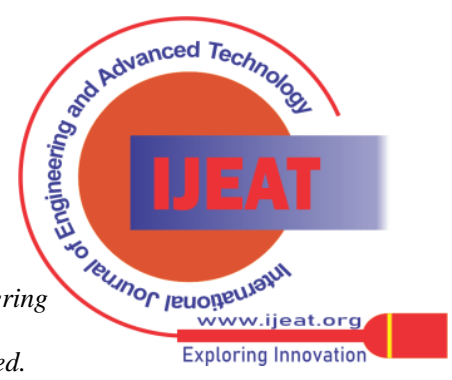




\section{AUTHORS PROFILE}

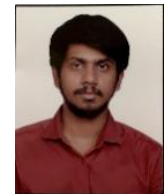

Yash Shah, UG Student, Mechanical Engineering, Indus University, Ahmedabad, Gujarat, India. Research work done in Design and Fabrication of Wheelchair cum Stretcher.

Neel Patel, UG Student, Mechanical Engineering, Indus University, Ahmedabad, Gujarat, India. Research work done in Design and Fabrication of Wheelchair cum Stretcher.

Nischay Patel, UG Student, Mechanical Engineering, Indus University, Ahmedabad, Gujarat, India. Research work done in Design and Fabrication of Wheelchair cum Stretcher.

Shail Patel, B.Tech, Mechanical Engineering, Indus University, Ahmedabad, Gujarat, India. Design and Development Engineer. Research work done in various domains of Mechanical Engineering.

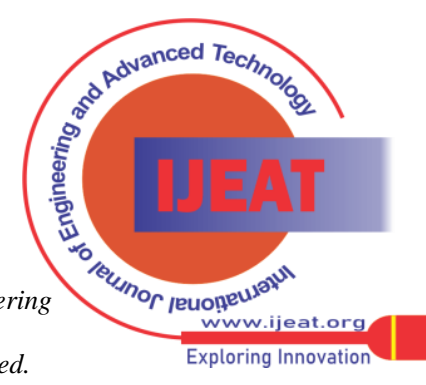

